\title{
LIGO End-to-End simulation Program
}

\author{
B. Bhawal*, G. Cella**, M. Evans*, S. Klimenko ${ }^{\dagger}$, E. Maros*, S.D. \\ Mohanty ${ }^{\dagger \dagger}$, M. Rakhmanov*, R.L. Savage*, jr., H. Yamamoto* \\ * LIGO Laboratory, California Institute of Technology, Pasadena, CA 91125, USA \\ ** Dipartmento di Fisica, Universita' di Pisa, piazza Torricelli 2, 56100 - Pisa, Italy \\ $\dagger$ Department of Physics, University of Florida, Gainsville, FL 32611, USA \\ t† Department of Physics, Pennsylvania State University, PA 16802, USA.
}

\begin{abstract}
A time-domain simulation program has been developed to provide an accurate description of interferometric gravitational wave detectors. This is being utilized to build a model of LIGO with the aim of aiding in the shakedown and integration of the interferometer subsystems, and ultimately the optimization of detector sensitivity.
\end{abstract}

Computer simulation is expected to play a crucial role in understanding and improving the performance of the LIGO interferometers. With that aim a simulation package with various modelling tools has been developed at Caltech. This is called LIGO End-to-End (E2E) simulation program [1].

The E2E package simulates the time-evolution of fields, optics, mechanical structures and electronic and control systems. It is written in $\mathrm{C}++$ and its modular design makes it possible to simulate wide variety of experimental configurations and processes using the same software without modifying the program. This package can be viewed as a software toolbox, like Matlab, and complex systems can be simulated by combining building blocks. The flexibility of the simulation environment makes it easy to add new physics or functionalities.

A graphical user interface, named Alfi, allows users to edit the description files for the simulation engine even for complex configurations in an easy way. Figure 1 shows the Alfi window for an example configuration of a Fabry-Perot (FP) cavity. For each module in the window (e.g., mirror, propagator, laser etc.), parameters can be set using the corresponding dialog box.

The evolution of optical fields and their interaction with optical media are calculated using the modal model [2]. The field is expanded using the Hermite-Gaussian eigenstate solutions of the paraxial wave equation. The E2E software can be used to simulate any planar optics configuration by combining mirrors and propagators without modifying the source code. This method is accurate and flexible but it suffers from speed penalty when the frequency of interest is much smaller than the inverse of the characteristic time-scale of the system. The simulation speed of

CP523, Gravitational Waves: Third Edoardo Amaldi Conference, edited by S. Meshkov (c) 2000 American Institute of Physics 1-56396-944-0/00/\$17.00 


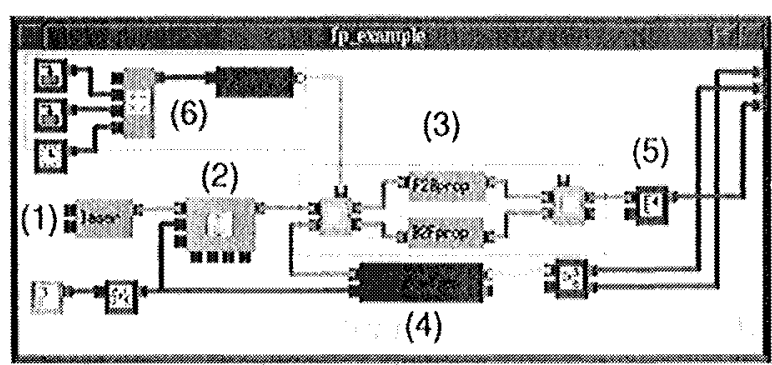

(a) GUI front-end of e2e

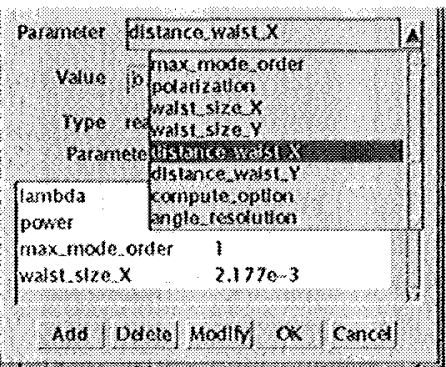

(b) parameter setting windows

FIGURE 1. Setup for Fabry-Perot cavity in E2E.

this kind of system can be improved by using approximate equations [3] of fieldevolution for writing a module corresponding to a composite system like a cavity. At present three kinds of cavities have been implemented using this technique: FP cavity, triangular cavity, recycled Michelson cavity.

The simulation of mechanical systems involves: seismic ground motion, seismic isolation and the suspension systems. The power spectra of the measured ground motion and the transfer functions of the isolation system can be parametrized and simulated using digital filters [4]. LIGO-I suspension system uses a single pendulum; A few models $[5,6]$ have been implemented and compared to validate each other. A new fully 3-D simulation code [6] called Mechanical Simulation Engine (MSE) is being implemented. This uses a basic set of fundamental objects (masses, beams etc.) which are combined to represent complex mechanical systems.

Modelling of the full 2-Km LIGO and its subsystems like Pre-Stabilized Laser and Input Optics and their validation are going on. Some open and closed loop transfer functions generated using this model are compared with measurements performed on the installed hardware and reasonable agreement is achieved.

Experiments on $2 \mathrm{Km}$ FP cavity at the LIGO Hanford Observatory began in November, 1999. The E2E software was used to study length control and lock acquisition. Also it was used to understand various higher order harmonic signals caused by the non-linear response of the optics systems in the in-lock state.

\section{REFERENCES}

1. Bhawal B., Evans M., Maros E., Rakhmanov M., Yamamoto, H., LIGO documents T970193, T970194.

2. Hefetz Y., Mavalvala N., Sigg D., JOSA, B14, 1597 (1997).

3. Redding D., LIGO document T960171; Bhawal B., JOSA, A15, 120 (1998).

4. Daw E., LIGO document T990112.

5. Mohanty S., LIGO document T990014; Rakhmanov M., Ph.D. theses (Caltech, 2000).

6. Cella G., LIGO documents T990106, T990107, T990108. 